\title{
25 Research Soure \\ Numerical Modeling of Rehabilitation Exercise when Distal Radius Fracture Treated by Splint
}

\section{Zhen Hua}

Wuxi Hospital Affiliated to Nanjing University of Chinese Medicine

Mingming Liu

The Second People's Hospital of Lianyungang

Mao Wu

Wuxi Hospital Affiliated to Nanjing University of Chinese Medicine

Bin Xie

Jiangyin Orthopaedic Hospital of Chinese Medicine

Yufeng Zhang ( $\nabla$ orthozhang@sina.com )

Wuxi Hospital Affiliated to Nanjing University of Chinese Medicine

Jianwei Wang ( $\nabla$ wangjianwei_1963@163.com )

Wuxi People's Hospital

Research

Keywords: Rehabilitation, bone fracture healing, elbow flexion exercise

Posted Date: June 18th, 2020

DOl: https://doi.org/10.21203/rs.3.rs-35435/v1

License: (c) (i) This work is licensed under a Creative Commons Attribution 4.0 International License.

Read Full License 


\section{Abstract \\ Background}

Rehabilitation exercise plays a key role in bone fracture healing. Although traditional clinical experiments and observations are adopted to evaluate the effect of rehabilitation exercise, the biomechanical mechanism is still unclear. There are few reports on the mechanical analysis model of elbow flexion rehabilitation exercise of the complete upper limb model previously. Because the upper limb structure is complex, the previous model is so simplified that soft tissue structure is not considered. It cannot reflect the change and distribution of the stress and stress state during elbow flexion exercise.

\section{Methods}

From the perspective of biomechanics, based on the CT scanning images of forearm for an aged female volunteer, a 3-D distal radius fracture forearm model is established. By introducing the soft tissues and the splint, a complete 3-D forearm model is finally reconstructed. The model includes ulna, radius, humerus, muscle, ligament, joint, wrist, skin and splint. The correctness of the model is verified by comparing the results of numerical simulation with the results of previous literature and clinical experiments.

\section{Results}

When the elbow flexion angle increases from 0 to 120 degrees, the stress increases from $0.21 \mathrm{Mpa}$ to $0.83 \mathrm{Mpa}$. When the elbow flexion angle increases from 0 to 120 degrees, the strain increases from 2$23 \%$, and the maximum strain at the fracture end is about $22.5 \%$. Also an analytical formula of the relationship fracture end strain and different elbow flexion angles is obtained by nonlinear fitting based on the simulation results, which is convenient for clinicians to predict fracture end strain.

\section{Conclusions}

During elbow flexion rehabilitation exercise, the stress of biceps brachii is large, and with the increase of elbow flexion angle, the stress shows an increasing trend. The stress in the area of distal radius fracture is also concentrated. Moderate stress concentration is helpful to the process of bone remodeling, so as to shorten the time of fracture healing. There was no obvious stress concentration in metacarpal bone and has little effect on the activity of metacarpophalangeal joint. The fretting strain at the fracture site can improve the speed of fracture healing, because the generation of early callus can absorb a lot of energy generated by fretting strain, which has an impact on the mechanical properties and plastic deformation ability of callus, and plays an important role in the stimulation of late osteogenesis. 


\section{Background}

Distal radius fracture (DRF) is the most common fracture of human upper limb, particularly in older people with osteoporosis ${ }^{[1-3]}$. The incidence rate of DRF is high, which accounts for $20 \%$ of all fractures seen in emergency departments ${ }^{[4-6]}$. Limb rehabilitation exercise after splinting fixation, especially elbow flexion, plays an important role in DRF healing process. It not only helps to increase muscle strength, but also continuously stimulates the functional change of bone structure ${ }^{[7-8]}$. This is because the bone is a living material, and the bone structure has the adaptability of mechanical environment. Fracture healing is the bone shaping process of continuous induction, adjustment, feedback and circulation of the bone system, and finally the bone reaches the normal state. In the past, the effect of rehabilitation exercise is evaluated mainly by clinical observation and experiment, the biomechanical mechanism of elbow flexion exercise is not completely clear ${ }^{[9-10]}$. In addition, the quantitative research on elbow flexion exercise process has not been carried out now, and the parameters of the exercise process have not been correlated with the strain index in the fracture gap, so the rehabilitation exercise plan is mainly based on subjective experience. Instead of focusing on the influence of holding posture, blind exercise can achieve the opposite effect. Therefore, how to quantitatively evaluate and select the optimal exercise mode or exercise parameters to achieve the ideal therapeutic effect, so as to replace the traditional empirical exercise methods, is of great significance for rehabilitation exercise during DRF healing.

In recent years, many researchers conducted a significant amount of study on distal radius fractures [11${ }^{15]}$ mainly through biomechanical tests on specimen. Nevertheless, due to different kinds of restrictive conditions, the physiological conditions are hard to be truly reflected in the experiment. Many experiments were performed in vitro condition ${ }^{[16-17]}$, which were quite different from the real case in vivo condition. Moreover, the cadaver specimen are unavailable or hard to obtain. Therefore, how to solve with this problem, that is, choose the research methods to replace the traditional experimental ways is imperative for the biomechanical experimental research.

Recently, advanced computational biomechanics method has been applied into the clinical research of medicine. The finite element method (FEM) is widely used in the orthopedics field ${ }^{[18]}$. Compared with traditional research methods, the FEM has many advantages such as low cost, short-time computation, high-precision simulation, repeatability and so on. More importantly, this method can simulate the physiological conditions that cannot be truly reflected through destructive experiments ${ }^{[19-20]}$. Many researchers have carried out numerical simulation of distal radius fracture ${ }^{[21-22]}$. In addition, some researchers study the dynamic characteristics of elbow flexion by establishing a simplified upper limb model ${ }^{[23-26]}$. There are few reports on the mechanical analysis model of elbow flexion rehabilitation exercise of the complete upper limb model previously. Because the upper limb structure is complex, the previous model is so simplified that soft tissue structure is not considered. It cannot reflect the change and distribution of the stress and stress state during elbow flexion exercise. 
In view of the existing problems in the previous studies, in this paper, a refined human upper limb musculoskeletal biomechanical model is established. The model includes ulna, radius, humerus, muscle, ligament, joint, wrist, skin and splint. Combined with the previous literature and experimental test, the mechanical material parameters of each structure are determined, the distribution of stress and strain of the structure in the process of elbow flexion after splinting fixation is simulated, and the relationship between the strain at the fracture gap and the angle of elbow flexion is analyzed, and the equation of the relationship between the parameters of rehabilitation exercise and the strain at the fracture end is established, which provides the possibility to obtain the optimal exercise parameters. The technical methods and research results of this paper provide the basis for further study on the micro mechanism of fracture healing.

\section{Results}

\section{Model calibration}

Based on the presented model with no splints, the stress distribution of the articular surface for the wrist joint was obtained according to the Load case 1, as shown in Figure 7. The stress is unevenly distributed with the maximum value approximates $9.4 \mathrm{MPa}$. In the previous literature ${ }^{[20]}$, the maximum contact stress of the joint surface of the wrist joint was about $10 \mathrm{MPa}$ and the stress was mainly concentrated on the ulnar and ulnar bones of lunate bone. By comparison, the difference of the maximum stress is only $6 \%$. Thus, the presented model is verified to be accurate.

In order to verify the accuracy of the finite element model with splints, the principal strain distribution in the area near the fracture section of the distal radius is obtained via numerical simulation according to the Load case 2, as shown in Figure 8 . It is shown that the strain in the fracture area of distal radius is within $10 \%$. It can be seen from the literature ${ }^{[34]}$ that at different stages of fracture healing, the fretting strain stimulation required by callus is different. At the early stage of fracture healing, the fretting strain at the fracture end is less than $10 \%$, and the callus is seen forming. When the fretting strain is more than $30 \%$, there will be no new bone formation. It follows that the range of strain in the early stage of fracture healing is within $10 \%$, and that in the late stage of fracture healing is within $30 \%$. In this paper, the model is simulated at the initial stage of fracture healing and the numerical simulation results are consistent with those of the literature ${ }^{[34]}$.

Furthermore, the effect of splint fixation is verified via clinical experiments. Eleven adult volunteers from the physical examination center of Wuxi Affiliated Hospital of Nanjing University of Chinese Medicine from January to July in 2018 were chosen to be observed. The treatment effects of the splint that used in the distal radius fracture are observed, as shown in Figure 9. There were no oppressive feeling of apophysis and thenar, and there were no local skin pressure mark in the splint after 1 weeks. In addition, elbow flexion and extension of the metacarpophalangeal joint were not affected all cases. Therefore, it can be seen that splint fixation has a good clinical effect. 


\section{Numerical simulation results of elbow flexion}

Based on the numerical model, the distribution of stress and strain is obtained when the elbow bends from 0 to 120 degrees, as shown in Figure 10 and Figure 11. In the figures, von Mises stress is selected as the stress index, unit is $\mathrm{N} / \mathrm{m}^{2}$, and principal strain is selected as the strain index, unit is 1 . Because we focus on the stress and strain in bone and muscle structures, simulation results of them are only listed with the elbow flexion angle being 0 degree, 30 degree, 60 degree, 90 degree and 120 degree.

\section{Discussion}

\section{Comparison of stress with different elbow bending angles}

When the arm is flexing the elbow, the biceps brachii acts as the active muscle (the primary muscle and retracts to the center. In the meanwhile, the triceps brachii is the antagonistic muscle, relaxing and extending. The movement of bone and joint is mainly accomplished by the cooperation of two muscles. In the process of elbow flexion, the stress of biceps brachii is increasing with the increase of elbow flexion angle. When the elbow flexion angle increases from 0 to 120 degrees, the stress increases from $0.21 \mathrm{Mpa}$ to $0.83 \mathrm{Mpa}$. The comparison of the maximum stress under different elbow bending angles is shown in Figure 12. In addition, the stress concentrated in the area of the distal radius fracture. Moderate stress concentration is helpful to the process of bone remodeling, thus shortening the time of fracture healing and improving bone density ${ }^{[7]}$. Due to the fixation of splint and bandage, the stress of radius, ulna, styloid process of ulna and wrist joint is relatively large and even. There is no obvious stress concentration in metacarpal bone and has little effect on the activity of metacarpophalangeal joint.

\section{Comparison of strain with different elbow bending angles}

The strain distribution are similar to stress distribution, and comparison of the maximum strain under different elbow bending angles is shown in Figure 13. The strain in biceps and triceps is large as well as the strain in the area near the distal radius fracture segment. With the increase of elbow flexion angle, the strain at the fracture end shows an increasing trend. When the elbow flexion angle increases from 0 to 120 degrees, the strain increases from $2 \%$ to $23 \%$, and the maximum strain in the whole elbow flexion process is within the range of $30 \%$. Both animal experiments and clinical application studies have found

that the fretting strain at the fracture site can improve the speed of fracture healing ${ }^{[34]}$, which may due to that the generation of early callus can absorb a large amount of energy generated by fretting strain, affecting the mechanical properties and plastic deformation ability of callus, thus playing an important role in the stimulation of late osteogenesis.

This paper focuses on the strain at the distal radius fracture. Through numerical simulation, as shown in Figure 14, with the increase of elbow flexion angle, the strain at the fracture end shows a non-linear increasing trend. When the elbow flexion angle is 120 degrees, the maximum strain at the fracture end is about $22.5 \%$. 
The elbow flexion angle is denotes as the variable $\theta$, the fracture end strain is taken as the variable $\varepsilon$, and the above nonlinear curve is fitted based on MATLAB software. Because the bone structure is assumed to be elastic in the simulation, the curves of loading and unloading stages in the process of elbow flexion from $0^{0}-120^{0}-0^{0}$ can be considered to be consistent. Therefore, the equation of the relationship between the fracture end strain and the flexion angle is fitted as follows:

$\varepsilon=0.2052 \cdot \sin (0.02442 \cdot \theta-0.2103)+0.13 \cdot \sin (0.05271 \cdot \theta+1.892)$

$+0.0668 \cdot \sin (0.07116 \cdot \theta+4.278)$

(3)

where $\theta$ is the elbow flexion angle, and $\varepsilon$ is the fracture end strain. The fitting relationship between the strain of fracture end and the flexion angle is compared with the numerical simulation results, as shown in Figure 15. The relationship between the strain of fracture end and the angle of elbow flexion is thus established.

\section{Relationship between elbow flexion rehabilitation exercise parameters and fracture end strain}

The former elbow flexion rehabilitation exercise plan mainly depends on the subjective experience of the clinician. Different exercise plans have different effects on the speed of fracture healing. Accurate and quantitative prediction of fracture end strain is helpful for the diagnosis and evaluation of fracture rehabilitation effect. The goal of this paper is to establish the relationship between elbow flexion rehabilitation exercise parameters and fracture end strain, so as to provide help for the diagnosis and rehabilitation evaluation of the affected limb and better formulating rehabilitation exercise plan. Through the above analysis, the relationship between elbow flexion angle and fracture end strain has been established. In order to introduce elbow flexion rehabilitation exercise parameters, this paper will establish a more general equation of elbow flexion angle.

The elbow flexion angle is represented by $\theta$, in degrees; the frequency of daily exercise is represented by $f$, in times; the time of each exercise is represented by $t_{2}$, in minutes; the time of each elbow flexion cycle is represented by $t_{1}$, in seconds; the time of each exercise interval is represented by $t_{3}$, in hours; the duration of exercise is represented by $\tau$, in seconds; the maximum elbow flexion angle is represented by $\Phi_{\text {max }}$, in degree. The above parameters are set in the exercise plan. By establishing the relationship equation between the parameters and elbow flexion angle in 24 hours a day, the equation is written as follows:$$
\theta= \begin{cases}\Phi_{\max }\left|\sin \left(\frac{2 \pi}{2 t_{1}} \tau\right)\right|, & 0 \leq \tau \leq t_{2} \\ 0 \quad, & t_{2}<\tau \leq t_{3} \\ \Phi_{\max }\left|\sin \left(\frac{2 \pi}{2 t_{1}}\left(\tau-t_{3}\right)\right)\right|, & t_{3}<\tau \leq\left(t_{3}+t_{2}\right) \\ 0 & \left(t_{3}+t_{2}\right)<\tau \leq 2 t_{3} \\ \Phi_{\max }\left|\sin \left(\frac{2 \pi}{2 t_{1}}\left(\tau-2 t_{3}\right)\right)\right|, & 2 t_{3}<\tau \leq\left(2 t_{3}+t_{2}\right) \\ 0 \quad & \left(2 t_{3}+t_{2}\right)<\tau \leq 3 t_{3} \\ \ldots & \\ \ldots & \end{cases}
$$ 
It can be further simplified as follows:

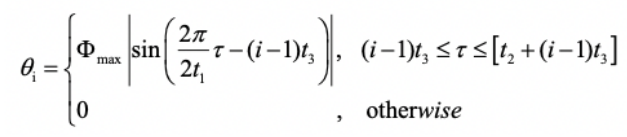

where, $\mathrm{i}$ represents the $\mathrm{i}$-th exercise, $0 \leq \mathrm{i} \leq \mathrm{f}$.

Herein, the relationship between elbow flexion rehabilitation exercise parameters and elbow flexion angle has been established. Combined with the relationship between fracture end strain and elbow flexion angle obtained by numerical simulation results fitting in equation (3), and substitutes equation (5) into equation (3), the relationship between elbow flexion rehabilitation exercise parameters and fracture end strain can be finally obtained, as follows:

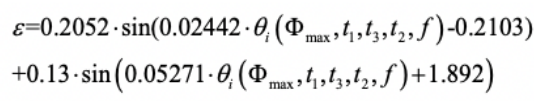

According to formula (6), it is very convenient for clinicians to predict the influence of different rehabilitation exercise parameters on fracture end strain through simple calculation, so as to make better rehabilitation exercise plan for patients.

\section{Conclusions}

Based on the CT data of the forearm of an elderly female volunteer, a new musculoskeletal biomechanical model of the human upper limb was reconstructed. The model includes ulna, radius, humerus, muscle, ligament, joint, wrist, skin and splint. The correctness of the model is verified by comparing the results of numerical simulation with the results of previous literature and clinical experiments. On the basis of this, numerical simulation of the stress and strain distribution of the human arm in the process of elbow flexion rehabilitation exercise are obtained and we focus on the stress and strain of muscles and bone fracture ends. The conclusion can be drawn as follows:

(1) During elbow flexion rehabilitation exercise, the stress of biceps brachii is large, and with the increase of elbow flexion angle, the stress shows an increasing trend. The stress in the area of distal radius fracture is also concentrated. Moderate stress concentration is helpful to the process of bone remodeling, so as to shorten the time of fracture healing. There was no obvious stress concentration in metacarpal bone and has little effect on the activity of metacarpophalangeal joint.

(2) With the increase of elbow flexion angle, the strain at fracture end increased. When the elbow flexion angle increases to 120 degrees, the strain is $23 \%$, and the maximum strain in the whole process of elbow flexion is within $30 \%$. The fretting strain at the fracture site can improve the speed of fracture healing, because the generation of early callus can absorb a lot of energy generated by fretting strain, which has 
an impact on the mechanical properties and plastic deformation ability of callus, and plays an important role in the stimulation of late osteogenesis.

(3) In this paper, an analytical formula of the relationship fracture end strain and different elbow flexion angles is obtained by nonlinear fitting based on the simulation results, which is convenient for clinicians to predict fracture end strain. On this basis, the rehabilitation exercise parameters are introduced, and a more general elbow bending angle equation is established. Through the results in this paper, it is convenient to predict the impact of different rehabilitation exercise parameters on the fracture end strain, so as to make a better rehabilitation exercise plan for patients.

The presented model can provide theoretical basis and technical guide for further investigating the fracture healing mechanism of distal radius fracture and clinical application of other splints. However, in this model, the materials of all the structures were assumed to be isotropic and with linear elastic properties. Actually, the soft tissue are anisotropic and viscoelastic. Thus, this can be improved in our future study.

\section{Methods}

\section{Geometry}

An aged female volunteer with $165 \mathrm{~cm}$ height and $55 \mathrm{~kg}$ weight is scanned using a 64-row spiral CT machine with the scanning layer thickness $0.625 \mathrm{~mm}$. Using the MIMICS 21.0 software, the 2D scanning data were segmented into different parts such as scapula, humerus, radius, ulna, carpus, metacarpus, phalanges, skin, soft tissue, biceps and triceps. The medical image data processing is shown in Figure 1.

The preliminary geometrical model is rough, which is a triangular patch model. There exists defects such as deformity, distortion and rough surface in the model. The STL file was imported into meshmixer software to repair the biceps and triceps models. The muscle start and stop points were set by stretching and polishing tools, and then the model was imported into 3 matic software to further optimize the surface mesh. The radius was cut about $3 \mathrm{~cm}$ away from the joint surface of the distal radius and divided into two segments to form the fracture model of the distal radius. The hand was selected from the soft tissue model of the arm palm to upper middle forearm surface mesh. The reconstructed geometrical model of forearm and fracture end model are shown in Figure 2 and Figure 3.

Two sets of splints $(150 \mathrm{~mm} \times 40 \mathrm{~mm} \times 8 \mathrm{~mm}$ and $120 \mathrm{~mm} \times 20 \mathrm{~mm} \times 8 \mathrm{~mm})$ were generated in 3matic. The surface mesh was cut from the level of thenar muscle to the upper middle part of forearm, and the sponge pad model was generated by an even outward offset of $2 \mathrm{~mm}$. The two sets of splints were moved and rotated according to the position of arm to match the arm. The model were then imported into the Geomagic studio 2014 software to repair and perform noise reduction, and finally form the geometrical model of arm with splint, as shown in Figure 4.

\section{Mesh}


The Hypermesh13.0 software was adopted to mesh the geometrical model. Adaptive mesh generation method was used to ensure the accuracy of calculation. In the model, tetrahedral elements were used to mesh the forearm. The total number of the elements is 373047 . The meshes are shown in Figure 5. In the software, the CONTACT element was used to define the contact pairs between the splint and the skin, the bone and the muscles, and the bone and the skin.

\section{Material properties}

All the structures are assumed to be elastic, isotropic and homogeneous. Based on published experimental data ${ }^{[27-32]}$, the elastic modulus of skin is $0.15 \mathrm{MPa}$ and the Poisson ration if 0.49 . The elastic modulus of muscle is $10 \mathrm{MPa}$ and the Poisson ratio is 0.45 . The elastic modulus of ligament is $0.3 \mathrm{GPa}$ and the Poisson ratio is 0.4. The elastic modulus of compact bone is $13.3 \mathrm{GPa}$ and the Poisson ratio is 0.3 . The elastic modulus of cancellous bone is $0.69 \mathrm{GPa}$ and the Poisson ratio is 0.3 . For obtaining the mechanical properties of the splint, the material mechanical test was conducted.

Table 1 Material properties of four willow splints

\begin{tabular}{ccccc}
\hline Group No. & No. 1 & No. 2 & No. 3 & No. 4 \\
\hline Elastic modulus (GPa) & 13.6 & 14.7 & 13.9 & 14.2 \\
\hline Poisson ratio & 0.27 & 0.31 & 0.33 & 0.28 \\
\hline Bending strength (GPa) & 10.9 & 8.69 & 10.83 & 9.88 \\
\hline The averaged elastic modulus (GPa) & \multicolumn{4}{c}{14.1} \\
\hline The averaged Poisson ratio & \multicolumn{4}{c}{0.30} \\
\hline The averaged bending strength (GPa) & \multicolumn{5}{c}{10.08} \\
\end{tabular}

In the test, four willow splints with the same size were selected. The splint was with a cuboid shape. The length was $153 \mathrm{~mm}$, the width $61 \mathrm{~mm}$ and the thickness $3 \mathrm{~mm}$. One end of the splint was fixed in three directions. The other end was loaded with a uniform force. Domestic WCD-50 displacement sensor was used to measure the displacement. Based on the cantilever beam model in material mechanics theory [33], the elastic modulus and Poisson ratio can be obtained, as shown in Table 1. The elastic modulus, Poisson ratio and bending strength were measured and calculated to be $14.1 \mathrm{GPa}, 0.3$, and $10.08 \mathrm{GPa}$ respectively.

\section{Boundary conditions}

The proximal clavicle and scapula are completely constrained in three coordinate directions. The contact pairs are set at the surface of bone, soft tissue and joint. The friction coefficient is $0.002{ }^{\text {[21] }}$. The contact pairs are set at the contact surface of splint and arm skin. The friction coefficient is $0.05^{[21]}$. Three different load cases are defined as follows:

(1) Load case 1

In order to verify the correctness of the finite element model established in this paper, the calculation conditions that consisted with the reference ${ }^{[20]}$ are selected, that is, a total of $100 \mathrm{~N}$ pressure load was 
applied in the second metacarpal bones and the third metacarpal bones along the hand and forearm with no splint.

\section{(2) Load case 2}

In order to verify the correctness of the finite element model after adding the splint, the calculation condition which is consistent with the bandage force tested in the clinical experiment is selected, that is, the bandage force load of $10 \mathrm{~N}$ is applied to the center of each splint.

\section{(3) Load case 3}

The whole elbow flexion process includes "straightening-bending-straightening". The flexion angle of forearm changes from " $0^{0}-120^{\circ}-0^{0}$ ", and the period of a cycle is assumed to be $t_{1}=6 \mathrm{~s}$. That is, it takes $3 \mathrm{~s}$ from the initial straightening state to the end of bending state. The exercise times $n_{1}=3$ in a day of 24hour and the time of each exercise is $t_{2}=10 \mathrm{~min}$. The total time of three exercise is $30 \mathrm{~min}$. The times of elbow flexion in each exercise is $n_{2}=100$, that is to say, the time of each exercise is 600 s. Assuming the elbow flexion angle is $\theta$, the relationship between elbow flexion angle and time is as follows:

$$
\theta=120^{\circ}\left|\sin \left(\frac{2 \pi}{T} t\right)\right|
$$

where $120^{\circ}$ represents the maximum flexion angle of elbow, $\mathrm{T}$ is $12 \mathrm{~s}, \mathrm{t}$ is time with unit $\mathrm{s}$. The equation (1) can be written as a more general equation shown follows:

$\theta=\Phi_{\max }\left|\sin \left(\frac{2 \pi}{T} t\right)\right|, \quad 0 \leq t \leq t_{1} \cdot n_{2}$

where $\Phi_{\max }$ represents the maximum flexion angle of elbow, $\mathrm{n}_{2}$ represents the number of times of elbow flexion in each exercise, $t_{1}$ represents the time of an elbow flexion motion, and the total time of one exercise is $t_{1} * n_{2}, t$ is the time, and the unit is $s$. The curve of elbow flexion angle with time is shown in Figure 6.

\section{Declarations}

\section{Ethics approval and consent to participate}

This study was compliant with ethical standards (K201903002).

\section{Consent for publication}

Written informed consent for publication was obtained from all participants.

\section{Competing interest}


The authors declare that they have no conflict of interest.

\section{Funding}

This study was funded by Science and Technology Project of Wuxi (CSE31N1514), Health and Health Committee Project of Jiangyin (S201909).

\section{Authors' contributions}

J. Wang, Z. Hua and M. Liu lead the conceptual design of the study, developed the numerical model and prepared and analysed the data. M. Wu and B. Xie participated in the establishment of the model. Y. Zhang participated in the analysis of the data. All authors read and approved the manuscript.

\section{Acknowledgements}

Z. Hua acknowledges the financial support of the Science and Technology Project of Wuxi (CSE31N1514) and Health and Health Committee Project of Jiangyin(S201909).

\section{Abbreviation}

Not Applicable

\section{Availability of data and material}

The datasets used or analysed during the current study are available from the corresponding author on reasonable request.

\section{References}

1. Arnander MW, Newman KJ. Fractures of the distal radius. Surg. 2006;24(12):429-32.

2. White NJ, Rollick NC. Injuries of the Scapholunate Interosseous Ligament: An Update. J Am Acad Orthop Surg. 2015;23(11):691-703.

3. Gunther SB, Lynch TL. Rigid internal fixation of displaced distal radius fractures. Orthopedics. 2014;37(1):34-42.

4. Harreld K, Li Z Iconography. Intramedullary Fixation of Distal Radius Fractures. J Am Chem Soc. 2004;126(9):2696-703.

5. Yang $\mathrm{S}$, Zhang $\mathrm{S}$. Relationship between malunion o distal radius fracture and functional recovery. J Orthopedic China. 2010;18(6):468-70.

6. Flinkkilä T, Raatikainen T, Kaarela O, Hämäläinen. M 2000 Corrective osteotomy for malunion of the distal radius. Arch. Orthop. Traum. Su. 120: 23-26.

7. Alessandro DC, Ivan G, Daria S. A review of recent developments in mathematical modeling of bone remodeling. J Eng in Med. 2019;2(3):1-9. 
8. Watson N, Haines T, Tran P, Keating JL. A comparison of the effect of one, three, or six weeks of immobilization on function and pain after open reduction and internal fixation of distal radial fractures in adults: A randomized controlled rrial. J Bone Joint Surg Am. 2018;100(13):1118-25.

9. Pooya $H$, Kathleen $D$, Eric W. What's new in pediatric orthopaedic trauma: the upper extremity. J Pediatr Orthoped. 2020;40(4):283-6.

10. Wade SM, Nesti LJ, Wind GG. The inverted free functioning gracilis muscle transfer for restoration of elbow flexion following delayed presentation or failed primary nerve reconstruction of upper trunk injuries. Tech Hand up Extrem Surg. 2020;24(1):26-31.

11. Çelik A, Kovacı H, Saka G. Numerical investigation of mechanical effects caused by various fixation positions on a new radius intramedullary nail. Comput Methods Biomech Biomed Eng. 2015;18(3):316-24.

12. Mouzakis DE, Rachiotis $G$, Zaoutsos $S$. Finite element simulation of the mechanical impact of computer work on the carpal tunnel syndrome. J Biomech. 2014;47(12):2989-94.

13. Howes MC, Cutting P, Thomas M. Best evidence topic report. Bet2. Splinting of buckle fractures of the distal radius in children. Emerg Med J Emj. 2008;25(4):222-3.

14. Burkhart TA, Andrews DM, Dunning CE. Multivariate injury risk criteria andinjury probability scores for fractures to the distal radius. J Biomech. 2013;46(5):973-81.

15. Matsuura Y, Kuniyoshi K, Suzuki T. Accuracy of specimen-specific nonlinear finite element analysis for evaluation of distal radius strength in cadaver material. J Orthop Sci. 2014;19(6):1012-8.

16. Khader BA, Towler MR. Common treatments and procedures used for fractures of the distal radius and scaphoid: A review. Mat Scieng C-Mater. 2017;74:422-33.

17. Dolce D, Goodwin D, Ludwig M, Edwards S. Intraoperative evaluation of dorsal screw prominence after polyaxial volar plate fixation of distal radius fractures utilizing the Hoya view: a cadaveric study. Hand. 2014;9(4):511-6.

18. Pfeiffer FM. The Use of Finite Element Analysis to Enhance Research and Clinical Practice in Orthopedics. J Knee Surg. 2016;29(2):149-58.

19. Goulding A, Jones IE, Taylor RW, Manning PJ, Williams SM. More Broken Bones: A 4-Year Double Cohort Study of Young Girls With and Without Distal Forearm Fractures. Journal of Bone Mineral Research. 2000;15(10):2011-8.

20. Yu B, Chen WC, Lee PY. Biomechanical comparison of conventional and anatomical calcaneal plates for the treatment of intraarticular calcaneal fractures - a finite element study. Comput Methods Biomech Biomed Engin. 2016;27:1-8.

21. Hua Z, Wang JW, Lu ZF. 2018 The biomechanical analysis of three-dimensional distal radius fracture model with different fixed splints. Techno. Health Care 1-13.

22. Alluri RK, Bougioukli S, Stevanovic M. A Biomechanical Comparison of Distal Fixation for Bridge Plating in a Distal Radius Fracture Model. J Hand Surg. 2017;42(9):748-56. 
23. Milanowicz M, Kędzior. K 2016 Multibody model of the human upper extremity for fracture simulation. Int. J. Occup. Saf. Ergon. 22(3): 320-326.

24. Khoo TA, Normahira M, Revati. R 2016 A finite element analysis of elbow joint in daily activities.J. Eng. Appl. Sci. 11(8): 5139-5145.

25. Adewusi S, Thomas M, Vu VH, Li W. Modal Parameters of the human hand-arm using finite element and operational modal analysis. Mech Ind. 2014;15(6):541-9.

26. Adewusi SA, Rakheja S, Marcotte P. Biomechanical models of the human hand-arm to simulate distributed biodynamic responses for different postures. Int J Ind Ergonom. 2012;42:249-60.

27. Gislason MK, Stansfield BW, Nash DH. 2010 Effects of partial wrist arthrodesis on loading at the radiocarpal joints. In: 17th Congress of the European Society of Biomechanics: 5-8.

28. Bajuri MN, Mohammed RA, Murali MR. Mechanical and functional assessment of the wrist affected by rheumatoid arthritis: A finite element analysis. Med. Eng Phy. 2012;11:1294-302.

29. Sebastian FB, Enrico DA, Patrick W. Assessment of a novel biomechanical fracture model for distal radius fractures. Bmc Musculoskel Dis. 2012;13:252.

30. Streekstra GJ, Braskamp P, Vander LC. Cartilage thickness measurement in the subillimeter range. In: Medical Image Computing and Computer-assisted Intervention. Berlin: Springer-Verlag; 2004. pp. 950-8.

31. Bae JY, Park KS, Seon JK. Biomechanical analysis of the effects of medial meniscectomy on degenerative osteoarthritis. Med Biol Eng Comput. 2012;50(1):53-60.

32. Mcqueen MM. Redisplaced unstable fractures of the distal radius. A randomised, prospective study of bridging versus non-bridging external fixation. J Bone Joint Surg Br. 1998;80(4):665-9.

33. Sivarasu S, Lazar M. Finite element analysis of a novel high flexion knee used in total knee arthroplasty. Int J Bioeng. 2009;4(1):2778.

34. Yu X, Zeng B. Fretting stress and indirect fracture healing. Foreign Med Orthopaedics. 2004;25(3):166-8.

\section{Figures}
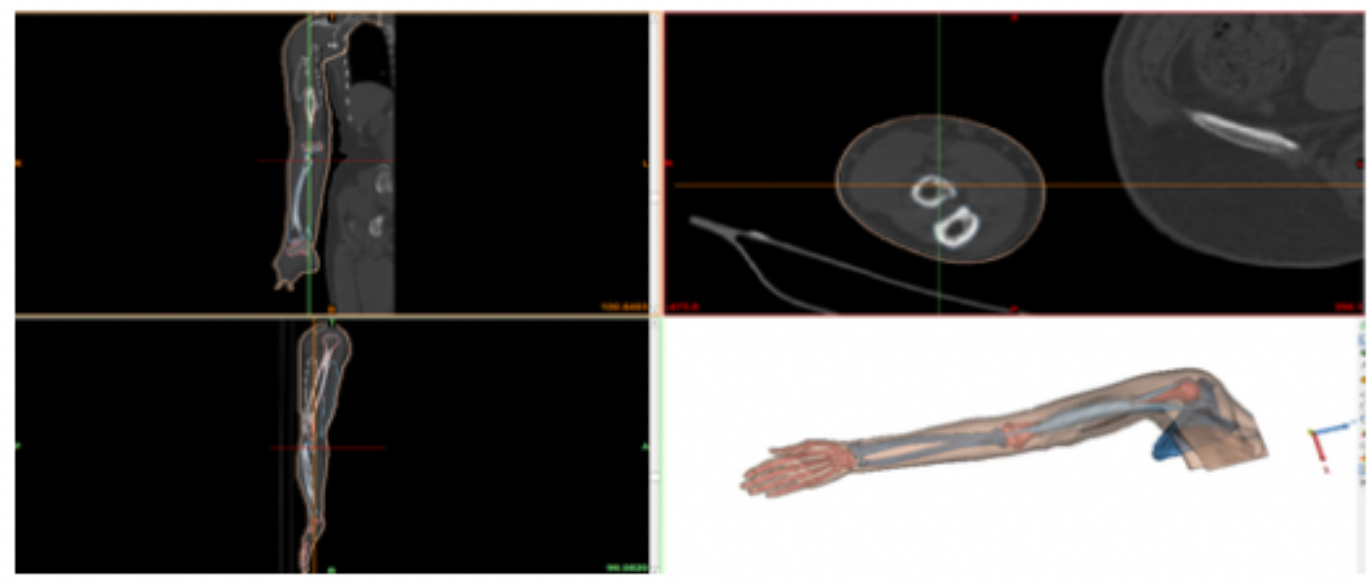
Figure 1

Preliminary 3D geometrical model of upper limb

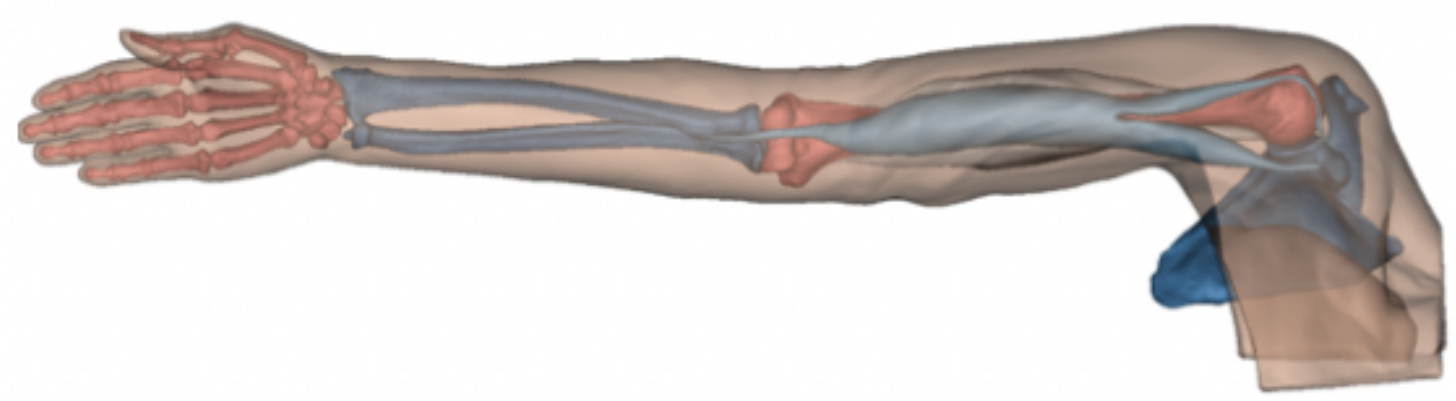

Figure 2

The 3-D integrated geometrical model of forearm including radius, ulna, wrist, muscle and skin

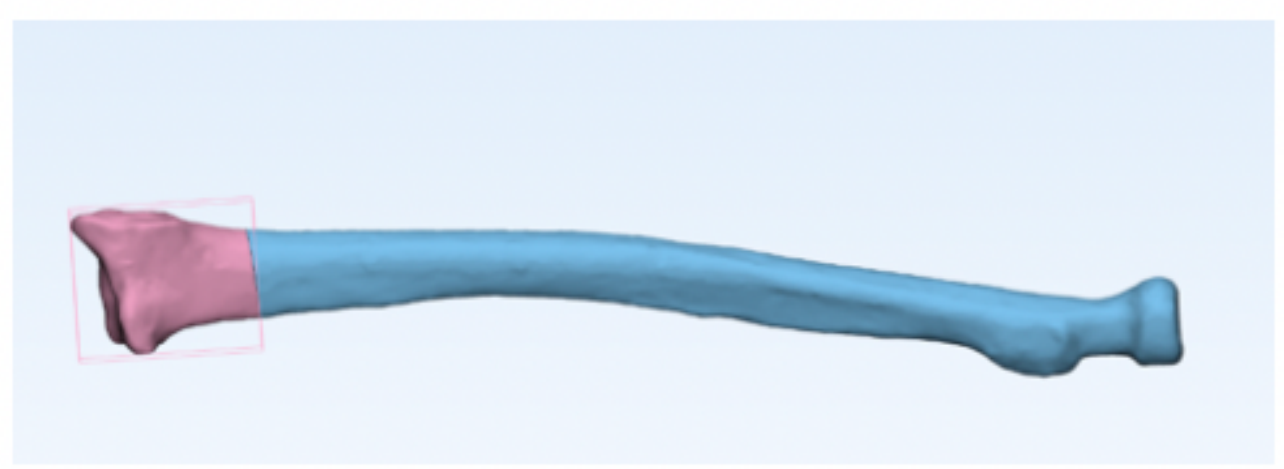

\section{Figure 3}

Fracture model of distal radius

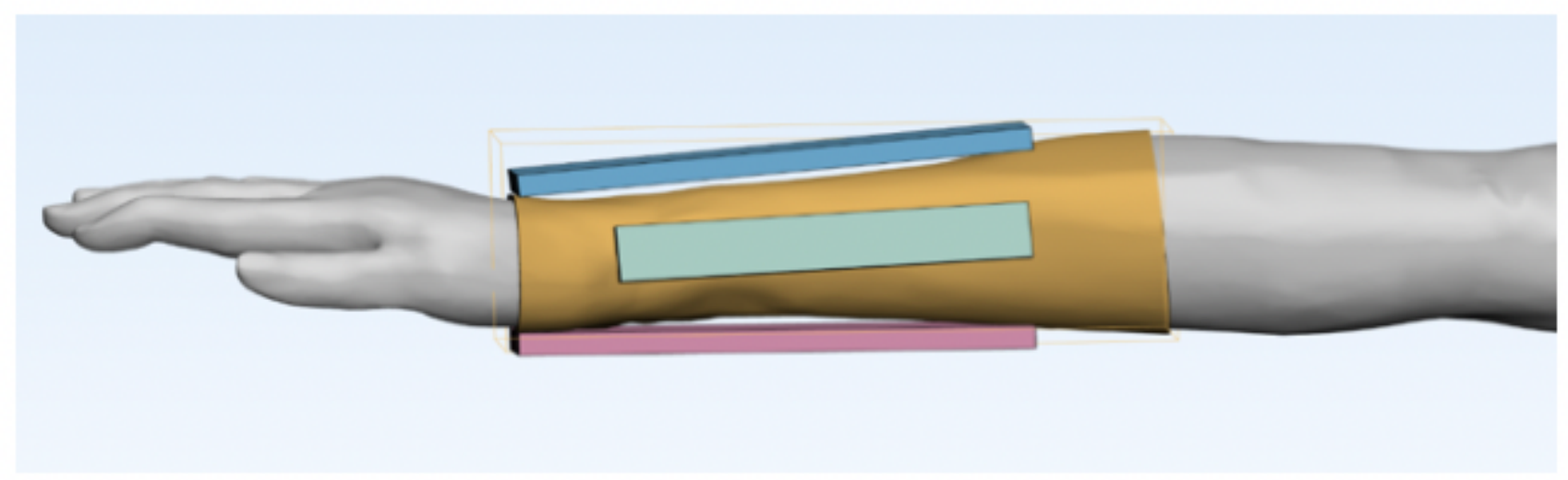

\section{Figure 4}

The finalized geometrical forearm model including willow splints 


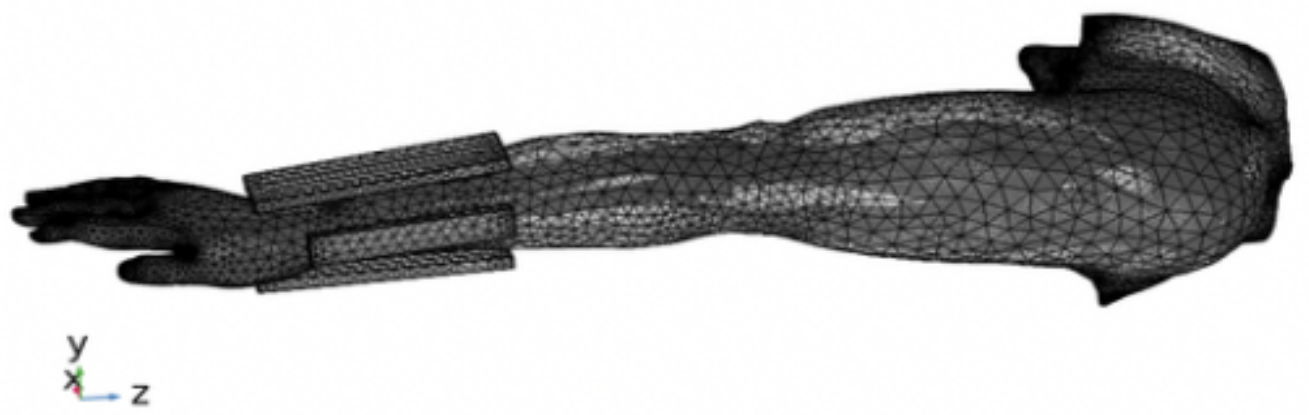

\section{Figure 5}

The mesh of model

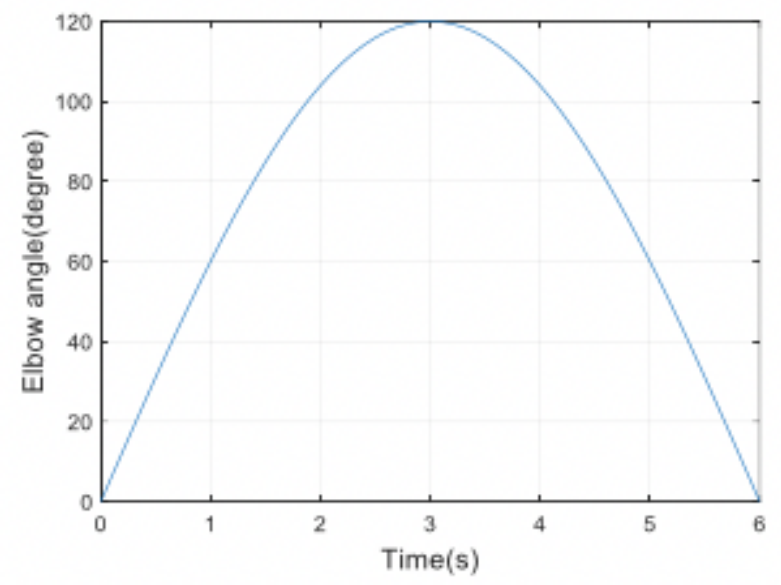

(a)

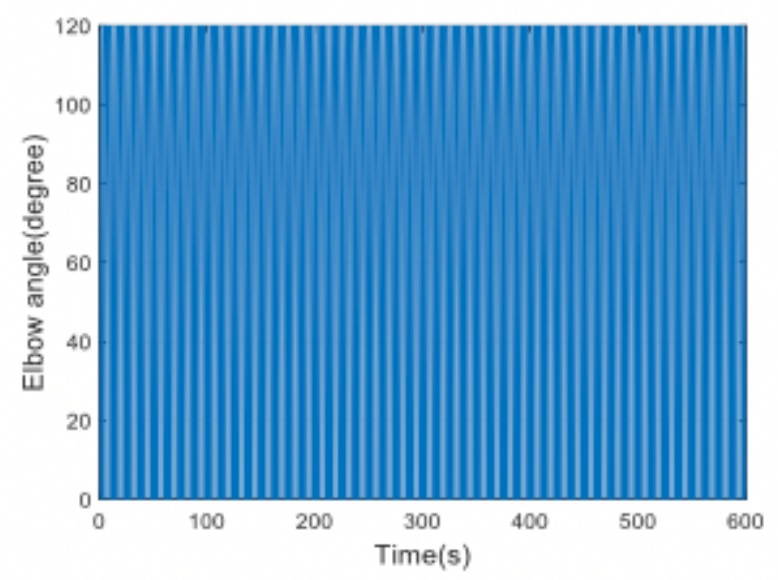

(b)

Figure 6

Variation of the angle of elbow flexion with time. (a) An elbow flexion motion; (b) A complete exercise process

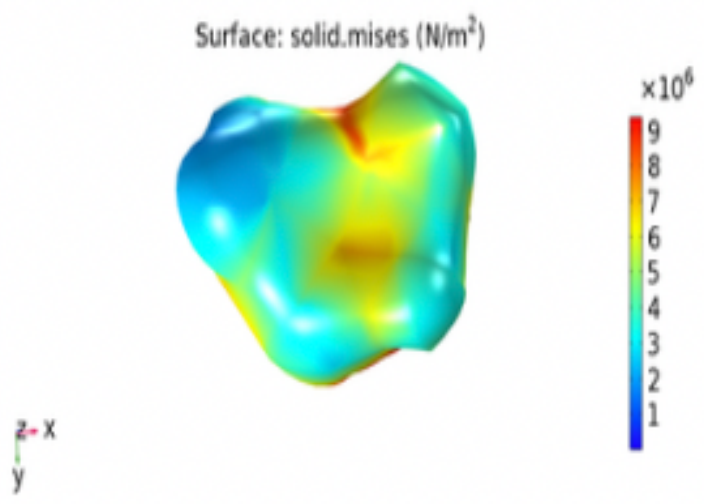

Figure 7 
Simulation stress results of the wrist joint surface

Sufface: First principal strain (N(m)
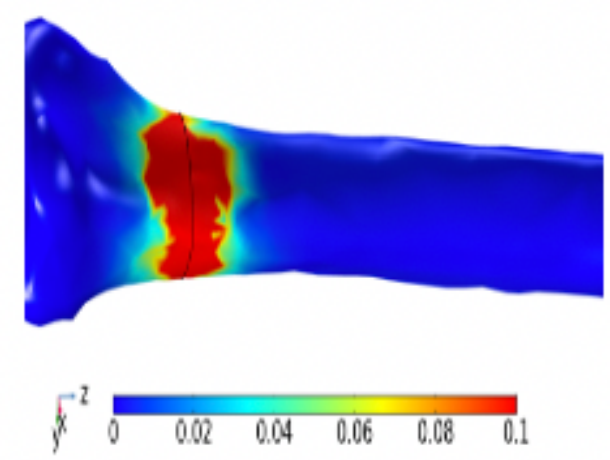

\section{Figure 8}

Principal strain of fracture area of distal radius

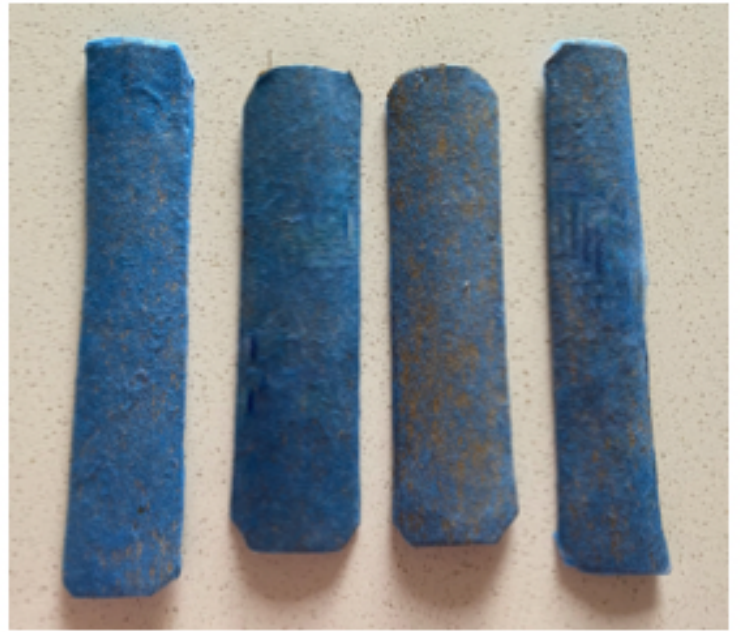

(A)

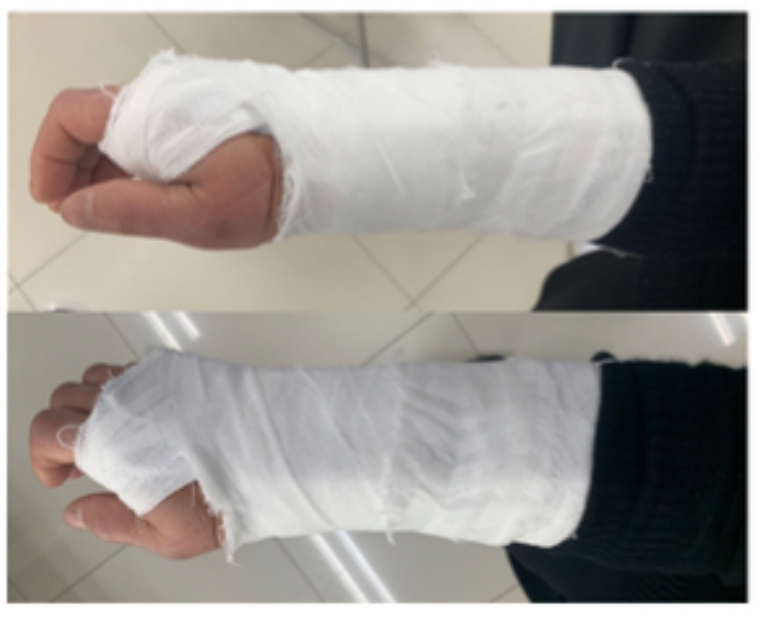

(B)

\section{Figure 9}

The observation of the limb fitting effect of the distal radius splint. (A. Splint; B. Metacarpophalangeal joint activity after suspension fixation) 


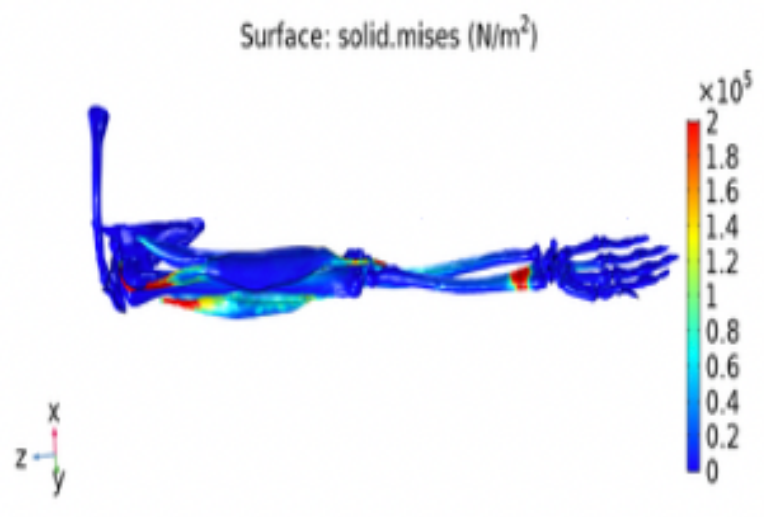

(a) Elbow flexion $\underline{\underline{0 \text { degree }}}$

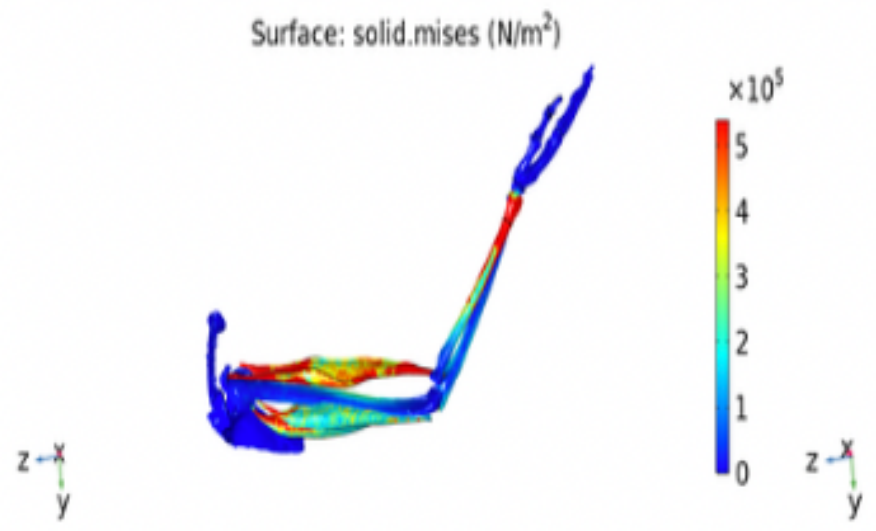

(c) Elbow flexion 60 degree

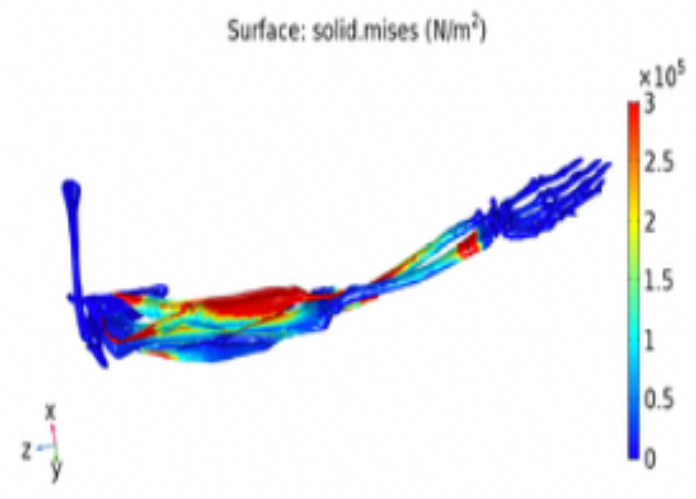

(b) Elbow flexion 30 degree

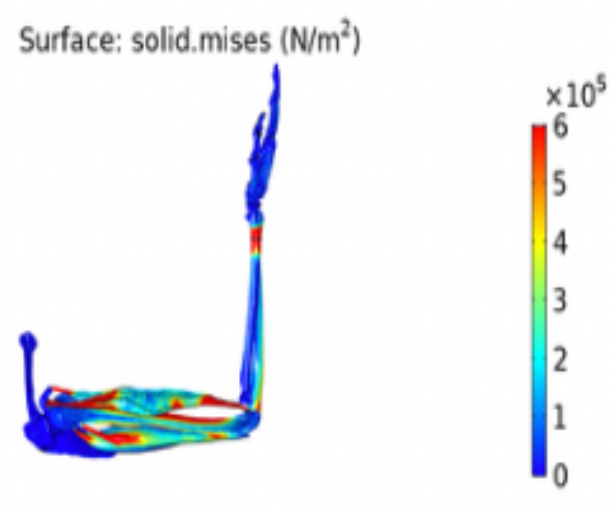

(d) Elbow flexion 90 degree

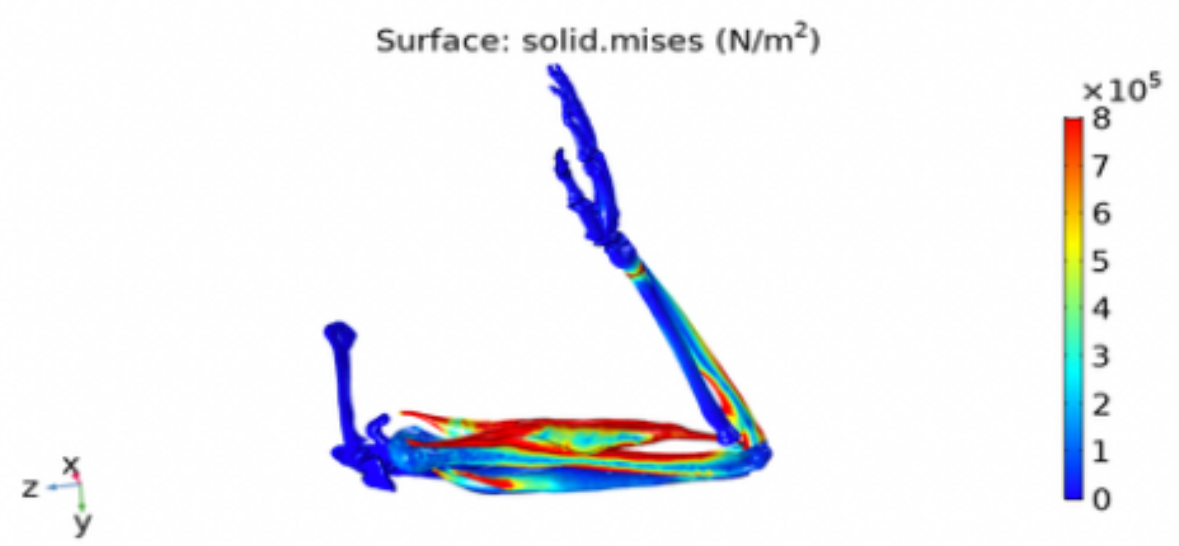

(e) Elbow flexion 120 degree

Figure 10

The stress distribution under different elbow bending angles of arm 


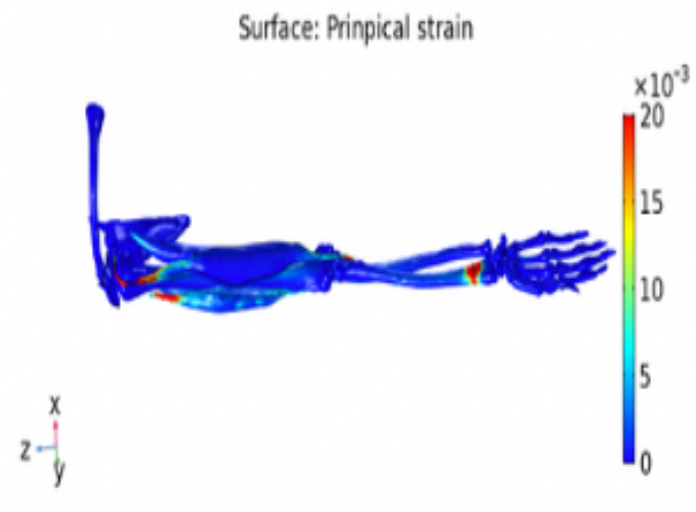

(a) Elbow flexion $\underline{\underline{0 \text { degree }}}$

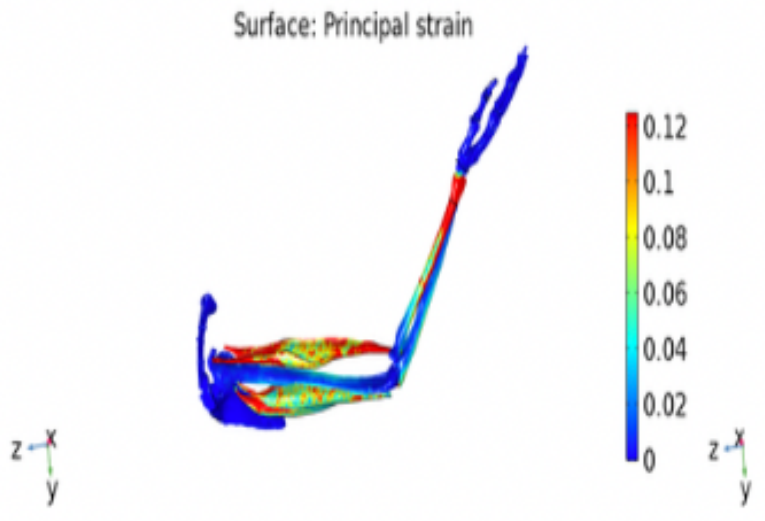

(c) Elbow flexion 60 degree
Surface: Principal strain

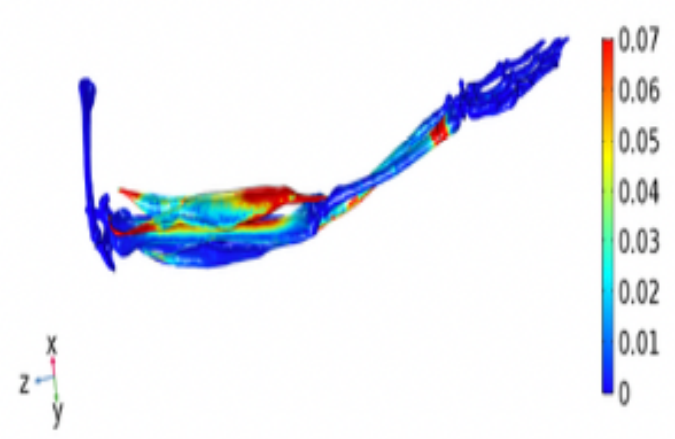

(b) Elbow flexion 30 degree

Surface: Principal strain

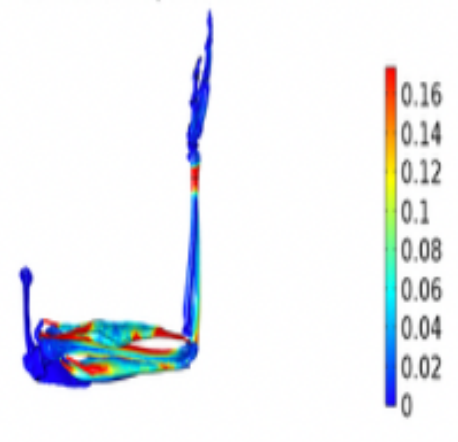

(d) Elbow flexion 90 degree

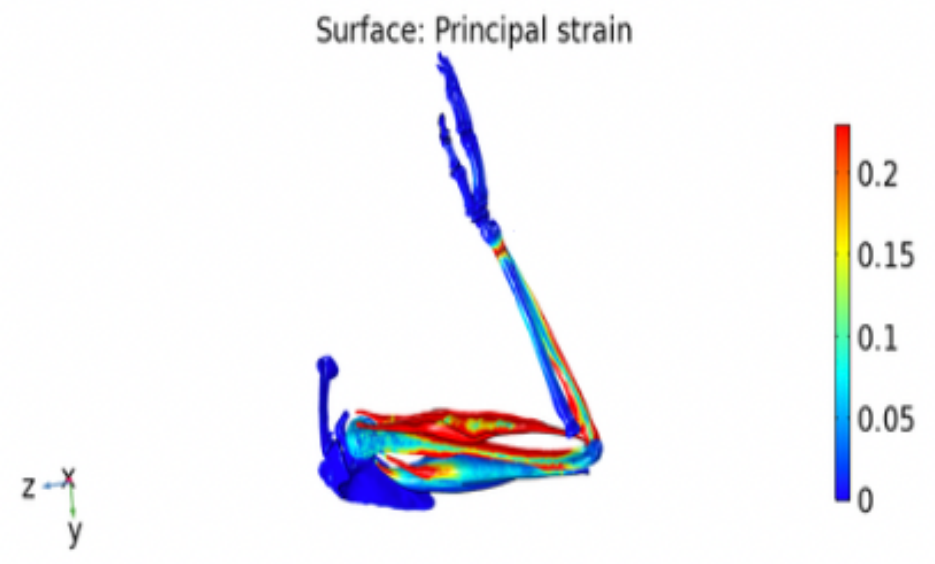

(e) Elbow flexion 120 degree

\section{Figure 11}

The strain distribution under different elbow bending angles of arm 


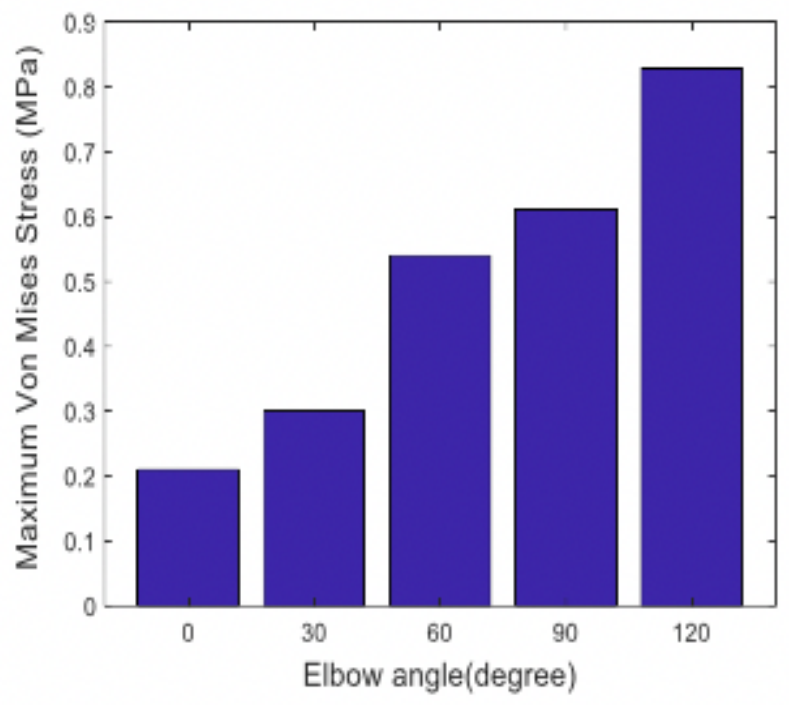

Figure 12

Comparison of the maximum stress for different elbow angle

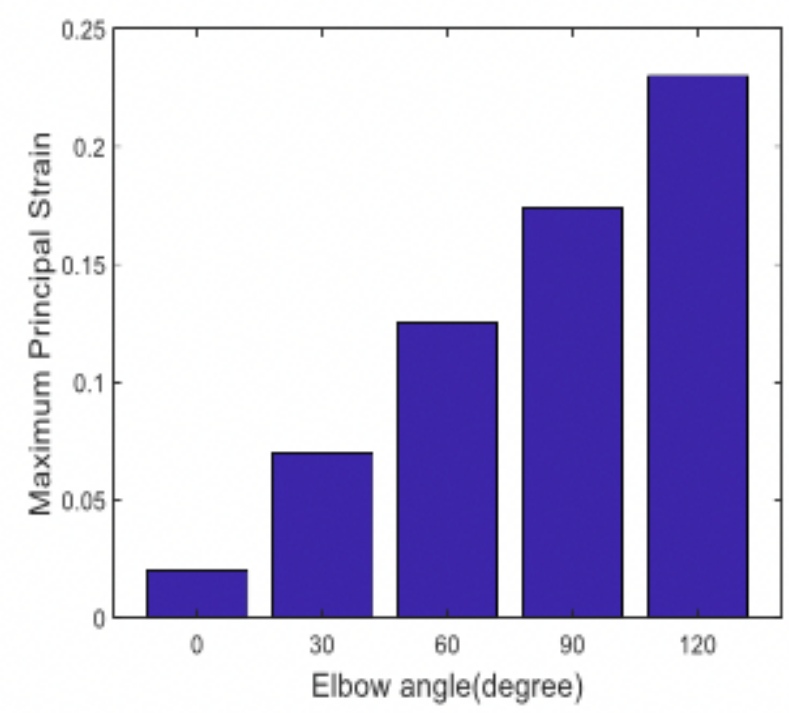

Figure 13

Comparison of the maximum strain for different elbow angle 


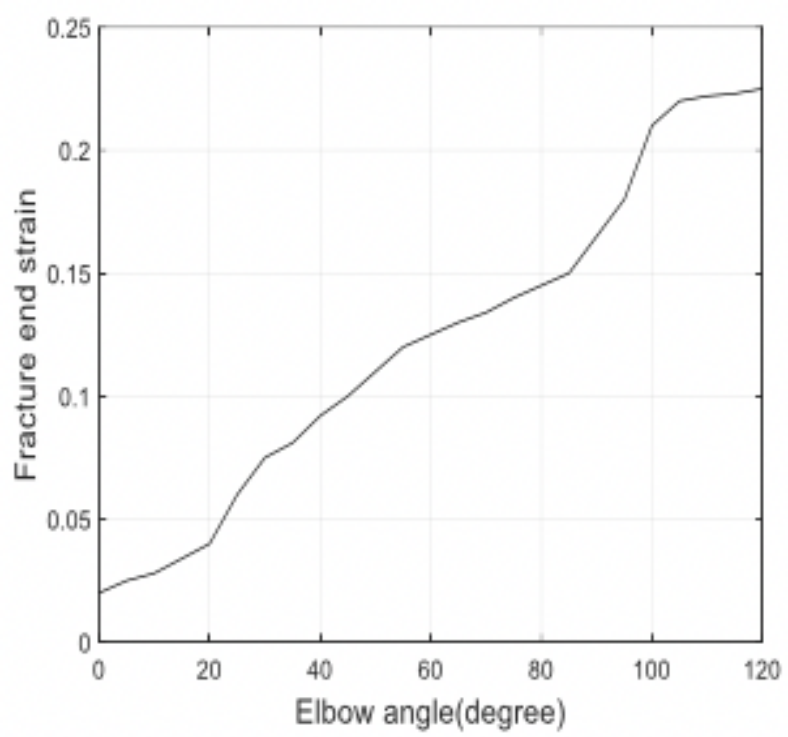

Figure 14

Variation of fracture end strain with different elbow angle

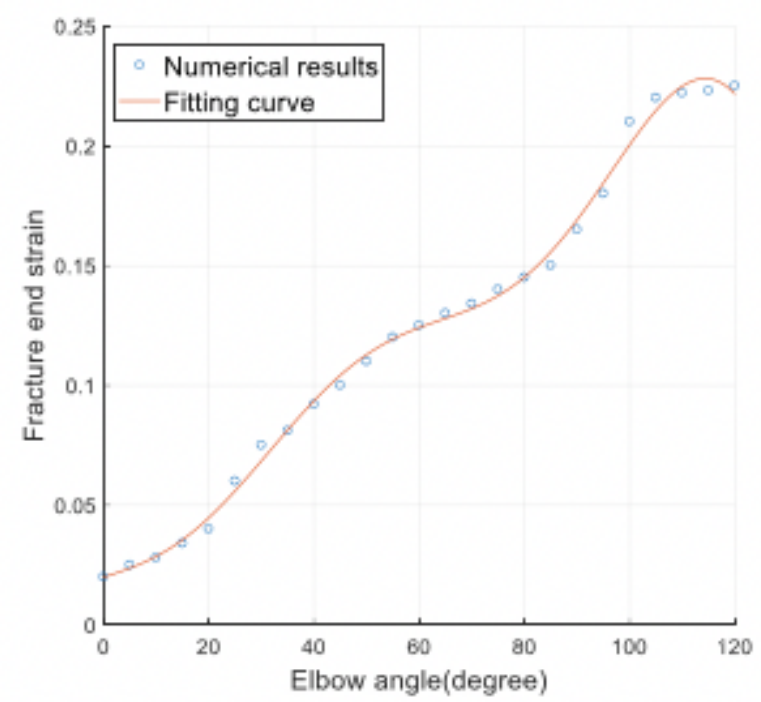

Figure 15

Comparison of numerical results with fitting curve data 